\title{
Active Particles Map to Passive Random Walks
}

\author{
Researchers make systems of self-propelled particles produce the same \\ large-scale dynamics as passive-particle systems.
}

\author{
By Rachel Berkowitz
}

$\int$ nder a microscope, a collection of active particles, such as bacteria moving in response to a chemical gradient, looks like a dynamic, out-of-equilibrium system. Zoom out, and the system can behave like a simple equilibrium system, experiencing only thermal motion and pairwise interactions. But what conditions make the two systems' dynamics indistinguishable on such scales? Jérémy O’Byrne and Julien Tailleur of the French National Center for Scientific Research (CNRS) and the University of Paris have answered this question by identifying corresponding sets of particle behaviors in active and passive systems that produce the same large-scale patterns [1]. Their results help explain how motile particles self-organize into different phases and could be used to design synthetic active matter.

Tailleur and O'Byrne modeled the microscopic dynamics of active particles whose speed and rotation are influenced by a chemical gradient. Then, they zoomed out to derive a description of the particles' motion on a larger scale. By

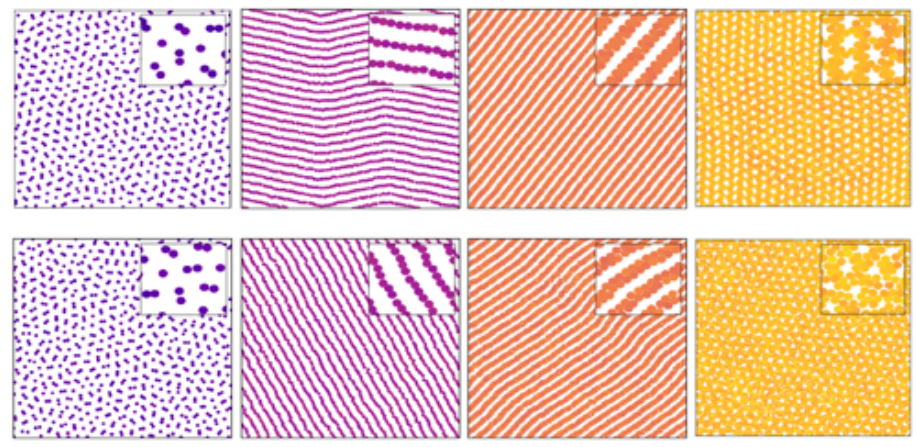

Credit: J. O'Byrne and J. Tailleur [1] tweaking the particle interactions, they deciphered parameters that produced large-scale motion indistinguishable from that of an equilibrium system. This established an unexpected mapping between disparate microscopic quantities-density measurements and interaction potentials, for example-in the active and passive systems. The researchers validated their mapping by comparing simulations of active and passive systems, showing that both could be made to produce identical spatial patterns.

Varying the initial particle density in the motile-particle simulations altered these large-scale patterns. In the equivalent equilibrium systems, such density changes mapped onto repulsive or attractive forces between particles. This method of controlling pattern formation could be used to manipulate active-matter systems in applications that call for coordinated behavior among particles without obvious neighbor-to-neighbor interactions.

Rachel Berkowitz is a Corresponding Editor for Physics based in Seattle, Washington, and Vancouver, Canada.

\section{REFERENCES}

1. J. O’Byrne and J. Tailleur, "Lamellar to micellar phases and beyond: When tactic active systems admit free energy functionals," Phys. Rev. Lett. 125, 208003 (2020). 